Published in final edited form as:

J Bone Miner Res. 2011 September ; 26(9): 2068-2081. doi:10.1002/jbmr.441.

\title{
An essential role for the association of CD47 to SHPS-1 in skeletal remodeling
}

\author{
Laura A Maile ${ }^{1, \ddagger},{ }^{,}$, Victoria E DeMambro ${ }^{2, \ddagger}$, Christine Wai $^{1}$, Ariel W Aday ${ }^{1}$, Byron E Capps ${ }^{1}$, \\ Wesley G Beamer ${ }^{3}$, Clifford J Rosen ${ }^{2}$, and David R Clemmons ${ }^{1}$ \\ Laura A Maile: Laura maile@med.unc.edu; Victoria E DeMambro: demamv@mmc.org; Christine Wai: \\ cwai@email.unc.edu; Ariel W Aday: ariel.aday@gmail.com; Byron E Capps: bcapps@email.unc.edu; Wesley G Beamer: \\ wesley.beamer@jax.org; Clifford J Rosen: crofen@gmail.com; David R Clemmons: endo@med.unc.edu \\ ${ }^{1}$ University of North Carolina at Chapel Hill, Division of Endocrinology \\ 2 Maine Medical Center Research Institute \\ 3 The Jackson Laboratory
}

\begin{abstract}
Integrin associated protein (IAP/CD47) has been implicated in macrophage-macrophage fusion. To understand the actions of CD47 on skeletal remodeling, we compared $C d 47^{-/}$with $C d 47^{+/+}$ controls. $\mathrm{Cd} 47^{-/}$mice weighed less and had decreased areal bone mineral density compared to controls. $C d 47^{-1-}$ femurs were shorter in length with thinner cortices and exhibited lower trabecular bone volume due to decreased trabecular number and thickness. Histomorphometry revealed reduced bone formation and mineral apposition rates, accompanied by decreased
\end{abstract}

\footnotetext{
*Address correspondence to: Laura A Maile, CB\# 7170, 5030 Burnett Womack, Division of Endocrinology, University of North Carolina, Chapel Hill, NC 27599-7170, Tel: (919) 966-2938, Facsimile: (919) 966-6025, laura_maile@ med.unc.edu.

*both authors contributed equally to this work

Manufacturers

AGFA

ALPCO

GE-Lunar

IDS

Life Technologies

Millipore Corporation.

Orthometrics

Peprotech Inc

Pierce.

Purina Mills International

Richard-Allan Scientific

Santa Cruz.

SAS

Scanco Medical AG

Sigma

Stoelting
}

All authors have no conflict of interest 
osteoblast numbers. No differences in osteoclast number were observed despite a non-significant, but $40 \%$ decrease, in eroded surface/bone surface in $C d 47^{-/-}$mice. In vitro the number of functional osteoclasts formed by differentiating $C d 47^{--}$bone marrow cells was significantly decreased compared to wild-type cultures and was associated with a decrease in bone resorption capacity. Furthermore, by disrupting the CD47-SHPS-1 association we found that osteoclastogenesis was markedly impaired. Assays for markers of osteoclast maturation suggested the defect was at the point of fusion and not differentiation, and was associated with a lack of SHPS-1 phosphorylation, SHP-1 phosphatase recruitment and subsequent dephosphorylation of non-muscle cell myosin IIA. We also demonstrated a significant decrease in osteoblastogenesis in bone marrow stromal cells derived from $\mathrm{Cd}_{47^{-/}}$mice. Our finding of cell autonomous defects in $C d 47^{-1}$ osteoblast and osteoclast differentiation coupled with the pronounced skeletal phenotype of $\mathrm{Cd}_{47^{--}}$mice support the conclusion that CD47 plays an important role in regulating skeletal acquisition and maintenance through its actions on both bone formation and resorption.

\section{Keywords}

bone; osteoclast; osteoblast; SHP-1; non-muscle myosin-IIA; bone resorption

\section{Introduction}

Bone remodeling is a physiological process dependent upon the balance between bone formation and bone resorption. This balance is maintained predominately by the number and activity of osteoblasts and osteoclasts. Osteoblasts are derived from mesenchymal stem cells while osteoclasts are derived from hematopoietic cells of the monocyte/macrophage cell line. As a single osteoclast (OC) differentiates, it expresses markers of the functional cell before it actually fuses. These pre-osteoclasts have the capacity to attach to bone and form a small ruffled border that begins the process of bone resorption. However, because of their small size and the relatively small volume of bone resorption enzymes, these single cells have limited functional capacity. Pre-osteoclasts must fuse into large OCs to have the surface area and enzymatic capacity to resorb significant quantities of bone. The coordination of osteoclastic differentiation with pre-osteoblast differentiation and elaboration of m-CSF and RANKL provide for a tightly coupled remodeling sequence.

A number of proteins have been identified as playing an important role in membrane fusion which must occur in order to form functional osteoclasts ${ }^{1}$. Integrin associated protein (IAP/ CD47) has been implicated in macrophage-macrophage fusion ${ }^{2}$. CD47 and Src homology 2 domain-containing protein tyrosine phosphatase (SHP) substrate-1 (SHPS-1/SIRPalpha/ MFR/BIT) are both members of the Ig superfamily of cell surface proteins. Direct protein binding and cell adhesion assays have shown that the extracellular domain of CD47 serves as a binding partner for SHPS-1 ${ }^{3,4-6}$. When CD47 binds to SHPS-1, SHPS- 1 can undergo tyrosine phosphorylation ${ }^{7}$. SHPS-1 tyrosine phosphorylation leads to recruitment of two phosphatases SHP-1 and SHP-2 that bind to SHPS- 1 through their SH2 domains ${ }^{8}$. SHP-1 recruitment to tyrosine phosphorylated SHPS-1 is required for SHP-1 activation in macrophages ${ }^{9}$. A recent report showed that an antibody that blocked SHPS-1-CD47 interaction resulted in decreased multinucleated osteoclast formation in vitro and that osteoclast number was reduced in $C d 47^{-/}$mice ${ }^{10}$. The role of CD47 in regulating osteoblast differentiation is less well defined. Therefore this study was undertaken to investigate the role of CD47 and its association with SHPS-1 in bone formation and resorption in vitro and in vivo. 


\section{Materials and Methods}

\section{Materials}

Alpha MEM medium, penicillin, and streptomycin were purchased from Life Technologies. Recombinant human soluble RANKL (shRANKL) was purchased from Peprotech Inc. Recombinant murine M-CSF was purchased from R and D Systems Inc. Polyvinyl difluoride membranes (Immobilon P) were from Millipore Corporation. Autoradiographic film was from Pierce. The monoclonal anti-phosphotyrosine (PY99) was from Santa Cruz. The anti-SHPS-1, SHP-1 and calcitonin receptor antibodies were purchased from Millipore Corporation. All other reagents were from Sigma unless stated.

The anti-CD47 monoclonal antibody, B6H12, was purified from a cell line derived from a B-cell hybridoma ${ }^{11}$. The anti-CD47 antibody (referred to as R569) is a polyclonal rabbit antiserum that recognizes amino acids 41 and 61 in the extracellular domain of CD47 12 . This antibody was used to detect intact CD47 via immunoblotting. The anti- $\beta 3$ antibody has been described previously 13 .

Mouse Husbandry-B6.129- $C d 47^{t m 1 F p l} / \mathrm{J}$ congenic strain, which had been backcrossed to C57BL/6J at least 10 times, was maintained in a research colony at The Jackson Laboratory. Mice were housed in groups of 4 or 5 of the same sex within polycarbonate boxes of $324 \mathrm{~cm}^{2}$ in area, on sterilized shavings of Northern White Pine. Colony environmental conditions included 14:10 hour light: dark cycles, with free access to acidified water ( $\mathrm{pH} 2.5$ with HCL to retard bacterial growth) and irradiated NIH-31 diet containing 6\% fat, $19 \%$ protein, Ca:P 1.15:0.85, plus vitamin and mineral fortified (Purina Mills International). All analyses were conducted using homozygous mutant mice $\left(\mathrm{Cd}^{-1-} 7^{-}\right)$ and same-sex littermate controls $\left(C d 47^{+/+}\right)$. For each experiment, the numbers of mutant and control mice used are provided. All experimental studies and procedures involving mice were reviewed and approved by the Institutional Animal Care and Use Committee of The Jackson Laboratory.

Sample collection for phenotypic studies-For bone phenotyping (DEXA, pQCT and MicroCT) groups of female and male $C d 47^{-1-}$ and $C d 47^{+/+}$control mice were necropsied and measured at 4, 8, 12 and 16 wks of age. For each mouse, body weight was recorded, whole body DEXA scans obtained, and tissue samples collected. Skeletal preparations were prepared as described previously ${ }^{14}$. Serum was harvested from whole blood collected at necropsy and stored at $-20^{\circ} \mathrm{C}$ until assayed.

\section{Dual-energy x-ray absorptiometry (DEXA) for areal bone mineral density} (aBMD) and body composition-DEXA scanning was performed using PIXImus (GELunar) to assess whole-body aBMD and body composition(lean mass and percent body fat) in both male and female $C d 47^{--}$and $C d 47^{+/+}$mice at $4,8,12$, and 16 wks of age. Bone mineral content by PIXImus is highly correlated with mineral content of hydroxyapatite standard of known density $\left(r^{2}=0.997\right){ }^{15}$. A phantom standard provided by the manufacturer was assessed each day for instrument calibration. Full body scans were obtained and X-ray absorptiometry data processed using Lunar PIXImus 2, vers. 2.1 software. The head was excluded from all analyses ${ }^{16}$.

\section{Peripheral quantitative computed tomography ( $\mathrm{PQCT}$ ) for volumetric bone} densitometry-pQCT was used to measure volumetric BMD (vBMD) on the left femur of female and male $C d 47^{-1-}$ and $C d 47^{+/+}$mice at 8 and 16 weeks. Isolated femur lengths were measured with digital calipers (Stoelting), and then femurs were measured for density using the SA Plus densitometer (Orthometrics). Calibration of the SA Plus instrument was 
accomplished with a manufacturer-supplied phantom and with hydroxyapatite standards of known density $\left(50-1000 \mathrm{mg} / \mathrm{mm}^{3}\right)$ with cylindrical dimensions $(2.4 \mathrm{~mm}$ diameter $\times 24 \mathrm{~mm}$ length). Accuracy of linear measures was checked with defined thickness aluminum foils. The bone scans were analyzed with thresholds of 710 and $570 \mathrm{mg} / \mathrm{cm}^{3}$, using Orthometrics software version 5.50 yielding cortical bone areas that were consistent with histomorphometrically derived periosteal values. Mineral content was determined with thresholds of 220 and $400 \mathrm{mg} / \mathrm{cm}^{3}$, selected so that mineral from most partial voxels $(0.07$ $\mathrm{mm}$ ) would be included in the analysis. Precision of the SA Plus for repeated measurement of a single femur was found to be within1.2-1.4\%. Isolated femurs were scanned at seven locations at 2-mm intervals, beginning $0.8 \mathrm{~mm}$ from the distal ends of the epiphyseal condyles. Total vBMD values were calculated by dividing the total mineral content by the total bone volume and expressed as milligrams per cubic millimeter. Cortical thickness was obtained at the midshaft scan ${ }^{16}$.

Microcomputed tomography-Distal trabecular bone volume fraction (BV/TV) and cortical architecture were measured by micro-CT (MicroCT40; Scanco Medical AG). This provides high-resolution data for trabecular bone volume as well as trabecular number, thickness, and spacing. Femurs from female and male $C d 47^{-1-}$ and $C d 47^{+/+}$mice at $16 \mathrm{wks}$ were scanned for micro architecture in the metaphyseal region of the distal femur. In addition, cortical thickness was obtained at the midshaft. The femurs were scanned at low resolution, energy level of $45 \mathrm{keV}$, and intensity of $177 \mu \mathrm{A}$. The distal trabecular scan started $0.6 \mathrm{~mm}$ proximal to the growth plate and extended proximally $1.5 \mathrm{~mm}$. One hundred fifty cross-sectional slices were made at $12-\mu \mathrm{m}$ intervals at the distal end beginning at the edge of the growth plate and extending in a proximal direction, and then 100 contiguous slices were selected for analysis. These were contoured inside the endosteal edge of the cortical shell to obtain the total volume (TV), followed by analysis of the trabecular bone volume (BV) with the Scanco software version 5.0. The scans for midshaft cortical thickness were obtained by 18 slices at the exact midpoint of the femur. These slices were contoured by user-defined thresholds for cortical bone and iterated across slices using the Scanco software.

Histomorphometry-Static and dynamic histomorphometry were performed by double labeling ${ }^{16-17}$ in the laboratory of Dr. Roland Baron. Male $C d 47^{-1-}$ and $C d 47^{+/+}$mice were injected (IP) with $20 \mathrm{mg} / \mathrm{kg}$ calcein at $16 \mathrm{wk}$ and again 7 days later. Mice were killed $48 \mathrm{~h}$ after the last injection. Muscle was removed and the femurs fixed in $10 \%$ buffered formaldehyde and rinsed in 70\% ethyl alcohol. Femurs were dissected and embedded in methyl methacrylate. Longitudinal sections, $5 \mu \mathrm{m}$ thick, were cut on a Micron microtome (Richard-Allan Scientific) and stained with $0.1 \%$ toluidine blue(pH 6.4). Static parameters of bone formation and resorption were measured in a defined area between 725 and 1270 $\mu \mathrm{m}$ from the growth plate using an Osteomeasure morphometry system(Osteometrics). For dynamic histomorphometry, mineralizing surface per bone surface and mineral apposition rate were measured in unstained sections under UV light. The terminology and units used are those recommended by the Histomorphometry Nomenclature Committee of the American Society for Bone and Mineral Research 18.

Serum analysis-Serum IGF-I levels were measured by an RIA (ALPCO) ${ }^{15}$. Serum osteocalcin was measured by IRMA (ALPCO) per the manufacturer's instructions. Serum TRAP5b was measured by ELISA (IDS) per the manufacturer's instructions. Serum Cterminal telopeptides of type I collagen $(\beta-\mathrm{CTx})$ and N-terminal propeptide of type I procollagen (PINP) levels were measured by EIA (IDS) per the manufacturer's instructions. The sensitivities were $0.01 \mathrm{ng} / \mathrm{ml}$ for IGF-I, $0.1 \mathrm{ng} / \mathrm{ml}$ for osteocalcin, $0.1 \mathrm{U} / \mathrm{L}$ for TRAP5b, 
$2.0 \mathrm{ng} / \mathrm{ml}$ for $\beta$-CTx and $0.7 \mathrm{ng} / \mathrm{ml}$ for PINP. The intra-assay coefficients of variation were between $3.7-9.2 \%$. All samples were analyzed within the same assay.

Osteoclast cultures-Bone marrow cells were isolated by flushing the femurs from 8 week old $C d 47^{-1-}$ and $C d 47^{+/+}$mice. Osteoclast-like cells were generated by plating the bone marrow cells at $1 \times 10^{6}$ cells per well in 96-well plates in differentiation medium: MEM supplemented with $5 \%$ fetal calf serum (FCS), M-CSF, ( $30 \mathrm{ng} / \mathrm{ml})$, and shRANKL $(30 \mathrm{ng} / \mathrm{ml})$. Media were changed at day 3 and day 6 . At day 7, the cells were fixed using $2.5 \%$ glutaraldehyde and stained for tartrate-resistant acid phosphatase (TRAP5b) (Sigma). TRAP5b-positive multinucleated (more than three nuclei)osteoclasts were counted using light microscopy. The number of TRAP5b-positive multinucleated cells was measured at predetermined sites of $5 \times 1 \times 1 \mathrm{~mm}$ squares in at least three wells per treatment and these results were expressed as mean \pm SEM. The results presented correspond to three independent experiments with at least three replicate wells within each experiment. Where indicated the anti-CD47 monoclonal antibody, B6H12, or an isotype matched control immunoglobulin (Con $\mathrm{IgG}$ ) was added to the differentiation medium at a final concentration of $5 \mu \mathrm{g} / \mathrm{ml}$. This was added each time the differentiation medium was refreshed e.g. every 3 days.

Pit formation assay-Bone marrow cells isolated as described above, were placed on top of dentin disks (IDS) one in each well of a 96-well tissue culture plate. The culture conditions were as above. After 14 days the dentin slices were washed with PBS, incubated in 5\% sodium hypochlorite for $10 \mathrm{~min}$, washed twice with water, and stained with $0.1 \%$ toluidine blue. The pits developed blue to purple color. Three disks were assessed per treatment, each in one well of a 96-well plate. Discrete resorption pits were counted by a blinded observer who scanned the entire surface of each disk with a reflected light microscope after staining.

\section{Expression of CD47 protein in bone marrow cells from $\mathrm{Cd} 47^{-/-}$mice-} Preparation of the plenti expression vector and virus stocks incorporating the cDNA encoding hemaglutinin (HA) tagged CD47 and the lacZ control (Con) has been described previously ${ }^{19}$. Bone marrow cells were isolated and plated in low attachment tissue culture plates and incubated in medium with M-CSF to enrich for macrophages. After 3 days the macrophages were plated on 96 well plates as described above were incubated with $100 \mu$ l of virus containing supernatant containing $4 \mu \mathrm{g} / \mathrm{ml}$ polybrene overnight. The virus supernatant was removed and the medium was replaced with differentiation medium and incubated for 7 days. Parallel cultures were stained for TRAP5b or lysed to analyze HA/CD47 expression by western immunoblotting.

Cell lysis and biochemical analysis-For the direct detection of protein in osteoclast/ osteoblast-like cells after 7 days in culture with M-CSF and RANKL cells were lysed in radioimmunoassay precipitation buffer (the lysate from three wells of duplicate treatments were pooled). Extracellular matrix preparations were made as we have described previously ${ }^{20}$. After centrifugation, equal amounts of cellular protein were mixed with nonreducing gelloading buffer, heated to $70^{\circ} \mathrm{C}$ for $10 \mathrm{~min}$, and separated by SDS-PAGE (8\%). To detected association between proteins or to analyze the tyrosine phosphorylation status immunoprecipitation studies were performed ${ }^{11}$. After SDS-PAGE, proteins were visualized by immunoblotting ${ }^{11}$. For immunoblotting, the antibodies were used at concentrations between 1:500 and 1:1000.

Osteoblast differentiation-Bone marrow cells were plated at $1 \times 10^{7}$ in 6-well tissue culture plates in -MEM with 10\% FCS. After 3 days the medium was changed, and the 
adherent cells were grown to day 7 . The media was then replaced with differentiation medium every two to three days till day 21 (a-MEM with 10\% FCS in the presence of 50 $\mu \mathrm{g} / \mathrm{mL}$ ascorbic acid and $10 \mathrm{mM}$ beta-glycerophosphate). At days 7, 14 and 21 adherent osteoblast progenitor cells were fixed with $4 \%$ paraformaldehyde and identified by alkaline phosphatase staining.

Alkaline phosphatase and von Kossa staining-Alkaline phosphatase (AP) activity was assayed based on the release of $p$-nitrophenol from $p$-nitrophenyl phosphate using an AP assay kit (Sigma). The number of AP-positive cells was counted using light microscopy. Five $1 \times 1 \mathrm{~mm}$ squares, in at least three wells, were measured per treatment and these results were expressed as mean \pm SEM. The cultures were then counterstained with von Kossa to detect mineral deposits at day 14 and 21.

Bone marrow mixed cell culture experiments-Whole bone marrow cells were plated at $1 \times 10^{6}$ in 96 well plates and treated with either vehicle, $\mathrm{PTH}\left(1 \times 10^{-7} \mathrm{M}\right)$ or 1,25 dihydroxyvitamin $\mathrm{D}\left(1 \times 10^{-8} \mathrm{M}\right)$ for seven to nine days at which point the cells were fixed with $2.5 \%$ glutaraldehyde and stained for TRAP5b positivity. Cells were then counted manually in each well with a criteria of $\geq 3$ nuclei considered to represent a mature osteoclast. The data represents three replicate wells per genotype per experiment with duplicate experiments performed.

Quantitative Real Time PCR-Total RNA was prepared from adherent bone marrow stromal cell cultures at Day 7 using the RNeasy Mini Kit (Qiagen). Four Hundred nanograms of RNA was then converted to cDNA in a reverse transcription reaction using the MessageSensor RT Kit (Ambion, Inc., Austin, TX) and random decamers as primers. The cDNA was then diluted 1:5 with water. Quantification of mRNA expression was carried out using an iQ SYBR Green Supermix in a iQ5 thermal cycler and detection system (BioRad). The geNorm reference gene selection kit (PrimerDesign Ltd., UK) was used to identify to the two best housekeeping genes for this experiment. Primers for Tnfsf11 (RANKL) and Tnfrsf11b(OPG) were purchased from PimerDesign and the expression of these genes was assessed by the $\Delta \Delta \mathrm{Ct}$ method. Primer sequences are available upon request.

MC3T3-E1 cell culture-Cultures of the subclone 4 (high osteoblast potential) and subclone 24 (low osteoblast potential) of the MC3T3-E1 cell line were maintained in alpha MEM medium with $10 \%$ fetal calf serum. Osteoblast differentiation was induced as above with ascorbic acid $(50 \mu \mathrm{g} / \mathrm{mL})$ and beta-glycerophosphate $(10 \mathrm{mM})$ for 14 days in 6 well plates prior to either lysis or alkaline phosphatase activity staining. Where indicated cells were incubated with thrombospondin (TS-1) at a final concentration of $1 \mu \mathrm{g} / \mathrm{ml}$ and/or $\mathrm{B} 6 \mathrm{H} 12$ at a final concentration of $1 \mu \mathrm{g} / \mathrm{ml}$.

Statistics-Statistical evaluation of skeletal phenotype was performed using the JMP ANOVA software program (SAS). The histomorphometry data were analyzed using Student's $t$ test. Chemiluminescent images obtained were scanned using a DuoScan T1200 scanner (AGFA) and band intensities of the scanned images were analyzed using NIH Image, version 1.61. The Student's $t$ test was used to compare differences between groups. Data are expressed as mean \pm SEM in tables and figures. Differences were considered significant when $p<0.05$. 


\section{Results}

\section{Loss of CD47 affects skeletal parameters}

Male $C d 47^{-1}$ mice had reductions in weight and lean body mass compared to $C d 47^{+/+}$ controls ( $\mathrm{p}<.05$ ) at all time points tested (Table 1). Female $C d 47^{-1-}$ mice exhibited no differences in body weight and lean muscle mass except at 12 weeks of age where body weight was slightly reduced ( $\mathrm{p}=0.05)$. No change in $\%$ body fat was noted in either gender. Total whole body areal bone mineral density (aBMD) was consistently and significantly less in the $C d 47^{-1}$ males and females versus $C d 47^{+/+}$controls at all ages (Table 1).

Femoral vBMD, by pQCT, was measured $(\mathrm{n}=12-30$ mice per genotype and gender) at 8 and 16 weeks of age (Table 2). Femur length was reduced in both male and female $\mathrm{Cd}_{4} 7^{-1-}$ mice compared to controls at 8 and 16 weeks $(\mathrm{p}<0.05)$. Total vBMD was significantly less in the $C d 47^{-/}$male and females compared to $C d 47^{+/+}$controls at both $8(\mathrm{p}<0.002)$ and 16 weeks $(\mathrm{p}<0.0001)$. In part this was related to markedly reduced cortical thickness noted in both genders at 8 and 16 weeks $(\mathrm{p}<0.0001)$. Neither periosteal or endosteal circumference was different in 16 week female $C d 47^{-1}$ mice, whereas in the males, these parameters were markedly reduced at both 8 and 16 weeks of age compared to $C d 47^{+/+}$controls. Cortical vBMD was also reduced in the null females at 8 and 16 weeks and in the null males at 16 weeks compared to controls. Distal trabecular bone volume fraction and cortical architecture were measured by MicroCT at 16 weeks for both males and females and their age matched $\mathrm{Cd} 47^{+/+}$controls. This method provides a bone volume fraction (BV/TV) as well as trabecular number, thickness and spacing. Analysis of the mid-shaft by MicroCT confirmed the reduced cortices exhibited by both genders of $C d 47^{-1}$ mice shown by pQCT.

Measurements of the distal femur revealed a $20-25 \%$ reduction in the trabecular bone volume fraction (\% BV/TV, $\mathrm{p}=0.01)$, and trabecular number ( $\mathrm{p} \unlhd 0.01)$ at 16 weeks in both male and female $C d 47^{-1-}$ mice compared to $C d 47^{+/+}$controls (Table 3 and figure 1). Both genders of $C d 47^{-1-}$ mice had reductions in trabecular thickness compared to $\mathrm{Cd} 47^{+/+}$ controls, however this was only significant in the female mice $(\mathrm{p}=0.03)$.

Histomorphometric analysis performed on 16 week male femurs confirmed that the distal trabecular bone volume fraction was reduced nearly $16 \%$ in $C d 47^{-/}$femurs $(\mathrm{p}<0.17)$ and this was associated with markedly reduced bone formation rates $(\mathrm{p}<0.001)$, osteoblast number $(\mathrm{p}<0.004)$, and mineral apposition rates $(\mathrm{p}<0.002)$ compared to $\mathrm{Cd}_{4} \mathrm{7}^{+/+}$controls (Table 4). No differences in osteoclast numbers were detected. However, eroded surface/ bone surface (ES/BS\%) a marker of osteoclast function although not significant due to measurement variability and small numbers of mice, was found to be reduced by $\sim 40 \%$ in the $C d 47^{-/}$male femurs compared to $\mathrm{Cd} 47^{+/+}$controls. Serum N-terminal propeptide of type I procollagen (PINP) a marker of bone formation was significantly reduced $(\mathrm{p}=0.03)$ at 12 weeks of age in the male $C d 47^{-1}$ mice compared to $C d 47^{+/+}$(Table 5) consistent with the histomorphometric findings. However $\beta$-CTx and TRAP5b levels were not different at 12 or 16 weeks. Insulin like growth factor-1 (IGF-I) serum levels were reduced $(p=0.03)$ at 8 weeks of age in the male $C d 47^{-1}$ mice compared to $C d 47^{+/+}$. However by sixteen weeks $C d 47^{-/-}$IGF-I levels were higher than $C d 47^{+/+}$controls $(\mathrm{p}=0.055)$.

\section{CD47 expression is required for functional osteoclast differentiation in vitro}

When bone marrow cells from male and female $\mathrm{Cd}_{4} 7^{-/}$and $\mathrm{Cd}_{4} 7^{+/+}$mice were cultured in M-CSF and RANKL, the numbers of TRAP5 $b^{+}$osteoclasts with $>3$ nuclei were significantly lower in the $\mathrm{Cd} 47$ null mice after 7 days compared to $\mathrm{Cd} 47^{+/+}$controls (figure 2). A representative image is shown in figure $2 \mathrm{~A}$. We detected almost no OC formation in either bone marrow cell cultures from either genotype treated with M-CSF alone. 
To determine if failure of OC formation was due to a direct lack of CD47 protein we expressed CD47 in the bone marrow cells derived from the $\mathrm{Cd} 47^{-1-}$ mice. Compared with cultures transduced with a control construct, RANKL stimulated a significant increase in OC formation in cells expressing CD47 to a level equivalent to that seen in bone marrow cells derived from wild-type mice (figure 2B compared with 2A). The level of CD47 expression achieved following viral transduction of the bone marrow cells is shown in figure $2 \mathrm{~B}$.

We also performed a bone resorption or "pit" assay to assess the activity of OCs from each genotype. A mean of $30 \pm 4$ pits/disk was detected following analysis of the $C d 47^{+/+}$wildtype bone marrow cells. In contrast a mean of $8 \pm 1$ pit/disk was detected following analysis of the $C d 47^{-/-}$bone marrow cells (mean \pm SEM $\mathrm{n}=3$ independent experiments with 3 disks/group in each experiment $* * p<0.05$ ). A representative image is shown in figure $2 \mathrm{C}$.

We compared the level of calcitonin receptor and $\beta 3$ integrin subunit (markers of osteoclast maturation expressed prior to fusion) in lysates from the day 7 cultures of the $C d 47^{-1-}$ and $C d 47^{+/+}$bone marrow cells. There was a significant increase in the amount of both the calcitonin and $\beta 3$ integrin subunit $(\mathrm{n}=3$ independent experiments $* * * p<0.01)$ in response to RANKL. This increase occurred in bone marrow cells from both $C d 47^{-1}$ and $C d 47^{+/+}$ mice and there was no significant difference in either the calcitonin receptor (figure $2 \mathrm{D}$; top panel) or $\beta 3$ integrin (figure 2D: middle panel).

These results suggest that the $C d 47^{-1}$ derived bone marrow cells were undergoing differentiation despite the lack of mature OCs; therefore we reanalyzed the TRAP5b staining data. When all the TRAP $5 b^{+}$cells were counted regardless of the presence of more than 3 nuclei, there was no significant difference in cell number between the two preparations (figure 2E). Assuming the difference between the total number of TRAP+ cells and TRAP+ with 3 nuclei reflects immature osteoclasts there should be fever immature osteoclasts in the cultures in which fusion has occurred. There were $(145 \pm 37)$ immature osteoclasts in the wild-type cultures compared with the CD47 deficient cultures (493 \pm 15 ; mean \pm SEM, $\mathrm{n}=$ $3 \mathrm{p}<0.01$ ). Taken together these data support the hypothesis that the lack of CD47 results in a lack of cellular fusion rather than cellular differentiation.

\section{Regulation of CD47 protein by RANK/RANKL}

We next examined the amount of CD47 present in bone marrow cells. The representative immunoblot shown in figure $3 \mathrm{~A}$ reveals that there is a significant increase in the amount of CD47 protein that can be detected in the lysate from the $C d 47^{+/+}$derived bone marrow cells that were analyzed following treatment with RANKL. There was no significant increase in the amount of CD47 in the lysates from the $C d 47^{-1}$ derived bone marrow cells after stimulation with RANKL (mean \pm SEM $n=3$ independent experiments $* * * p<0.01$ ).

To verify that the lack of osteoclastogenesis in the bone marrow cells derived from the $C d 47^{-1-}$ mice was not due to a lack of a receptor, we immunoblotted for RANK. There was no significant difference in the amount of RANK after 7 days of exposure to RANKL when the two different preparations of bone marrow cells were compared (figure 3B).

\section{CD47 regulates osteoclastogenesis through its association with SHPS-1}

Many of the effects of CD47 require its association with SHPS-1 therefore, we examined the effect of the anti-CD47 antibody (B6H12) which disrupts the association between CD47 and SHPS- ${ }^{19}$. When this antibody was added to bone marrow cells from $\mathrm{Cd} 47^{+/+}$treated with M-CSF and RANKL there was a significant, $5 \pm 0.7$ fold, reduction in TRAP5b ${ }^{+}>3$ nuclei osteoclasts [mean \pm SEM $\mathrm{n}=3$ independent experiments $(* * *<<0.001)$ figure $4 \mathrm{~A}$ ]. However, when all TRAP5 $\mathrm{b}^{+}$cells were counted regardless of the number of nuclei there 
was no significant effect of the anti-CD47 antibody (figure 4B). The anti-CD47 antibody had no effect on the total amount of CD47 protein that was detected.

We compared SHPS-1 phosphorylation in the lysates from the $C d 47^{-/-}$and $C d 47^{+/+}$cultures after 7 days stimulation with M-CSF and RANKL. While robust SHPS-1 phosphorylation was detectable in the lysates from the $C d 47^{+/+}$cultures, SHPS-1 phosphorylation was undetectable in the lysates from the $C d 47^{-/}$mice (figure 5A). Phosphorylation of the tyrosine residues in the cytoplasmic domain of SHPS-1 is required for the recruitment and activation of the tyrosine phosphatase SHP- $1^{8}$. Given the lack of SHPS- 1 phosphorylation in the lysates from the $C d 47^{-1}$ derived bone marrow cells we examined the recruitment of SHP-1 to SHPS-1. There was a significant increase in SHP-1 association with SHPS-1 in the lysates from the $C d 47^{+/+}$cultures following RANKL stimulation (figure 5A). In contrast there was no detectable SHP-1 recruitment to SHPS-1 in the $C d 47^{--}$derived bone marrow cells (figure 5A).

The recruitment and activation of SHP-1 is required for the dephosphorylation of nonmuscle myosin IIA which has been implicated as a regulator of macrophage fusion ${ }^{9}$. We thus compared the tyrosine phosphorylation status of non-muscle myosin IIA in the lysates from the wild-type and knockout bone marrow cells. In the day 1 cultures there was an equal amount of non-muscle myosin IIA phosphorylation that could be detected (figure 5B). However, by day 7 while there was a significant decrease in the non-muscle myosin IIA phosphorylation in the $C d 47^{+/+}$cultures, non-muscle myosin IIA remained highly tyrosine phosphorylated in the bone marrow cells from the $C d 47^{-1}$ mice (figure 5B). Furthermore, RANKL addition was required to detect non-muscle myosin dephosphorylation.

\section{CD47 is required for osteoblastogenesis in vitro}

Due to the defect in bone formation noted on histomorphometry in the $C d 47^{-1-}$ mice, we examined osteoblast proliferation and differentiation in vitro of bone marrow stromal cells from $C d 47^{-1}$ and the $C d 47^{+/+}$mice by alkaline phosphatase and von Kossa staining. Both measures of osteoblastic activity, as well as cell number were significantly reduced in the $C d 47^{-1-}$ cultures compared with controls at day 7, day 14 and day 21 (Figure 6A). To determine if the osteoclast defect was cell autonomous or related to the in vitro osteoblast defect, we then examined the expression of RANKL and OPG in day 7 bone marrow stromal cell cultures (Figure 6B). RANKL mRNA expression was significantly greater (2 fold, $\mathrm{p}=0.005$ ) in the $C d 47^{-/}$osteoblasts compared to $C d 47^{+/+}$cells while OPG mRNA was reduced by $20 \%$ ( $\mathrm{p}=0.005$ ) in the null cells compared to wildtype. The RANKL/OPG ratio was increased 2.5 fold in the $C d 47^{-/}$compared to $C d 47^{+/+}$cells. Thus despite the impairment in osteoblast differentiation, production of RANKL was not affected.

To further confirm that the osteoclast defect was independent of the osteoblast defect, we then studied bone marrow cultures containing pre-osteoblasts and pre-osteoclasts treated with PTH or vitamin D to stimulate RANKL production. We found a persistent reduction (i.e. 38\%) in TRAP5b staining osteoclasts in these mixed cultures from $\mathrm{Cd}_{4} 7^{-/}$cells compared to wild type despite the increase in RANKL expression found in the $C d 47^{-1-}$ cells (figure 6C).

To provide additional evidence for the association between CD47 expression and osteoblast maturation we analyzed CD47 protein in cultures of two subclones of the MC3T3 calvarial osteoblast cell line. We chose these two cell lines as they are reported to differ significantly in their ability to undergo osteoblastogenesis ${ }^{21}$ (ATCC numbers CR1-2593 and 2595). Our analysis of alkaline phosphatase activity in cultures of the two clones following 14 days incubation in differentiation medium confirmed that there was a significant difference in the differentiation potential of these cells (figure 6D). We also analyzed CD47 protein levels in 
cultures following 5 and 14 days incubation in differentiation medium. After day 5 there was almost no intact CD47 protein in the cultures from either clone although there was more in the lysate from the Clone 4 cultures. By day 14 there was a significant $3.9 \pm 0.7$ fold increase in intact CD47 that was detectable in the clone 4 lysate whereas the Clone 24 lysate showed no change (mean \pm SEM $n=3$ independent experiments; $p<0.001$ ) (figure $6 E$ ).

To provide more evidence that $\mathrm{CD} 47$ plays a role in osteoblastogenesis we determined the response to increasing the concentration of CD47 in the Clone 24 cells. We have shown that the extracellular matrix protein, thrombospondin -1 (TS-1) has a direct effect on the level of intact CD47 by protecting it from degradation by the protease MMP- $2{ }^{20}$. We examined TS-1 levels in the extracellular matrix of Clone 4 and 24 cells and determined that while there was detectable TS- 1 in the Clone 4 cells (high osteoblast potential/robust CD47 expression) the level of TS-1 in the extracellular matrix from Clone 24 was undetectable (Figure 6F top panel). Given that TS- 1 can inhibit CD47 degradation ${ }^{20}$ we examined whether adding TS- 1 to the Clone 24 cultures would increase intact CD47. The addition of TS- 1 to the Clone 24 cultures markedly increased CD47 protein (Figure 6F, lower panel). This increase was associated with an increase in SHPS-1 phosphorylation, which we have shown previously is dependent upon SHPS- 1 being able to associate with CD47 ${ }^{19}$. To determine that the increase in SHPS-1 phosphorylation was due to the increase in its association with CD47 we examined the effect of the anti-CD47 antibody that disrupts the association between CD47 and SHPS- ${ }^{19}$. In the presence of this antibody the $3.2 \pm 0.3$ fold increase in SHPS-1 phosphorylation induced in the presence of thrombospondin was completely inhibited (Figure 6G). Importantly, the addition of TS-1 was sufficient to permit osteoblastogenesis in the Clone 24 cells and the CD47 dependence of this response was demonstrated by the loss of osteoblast differentiation in the presence of the anti-CD47 antibody that blocks CD47 association with SHPS-1 (Figure 6H).

\section{Discussion}

This study shows that CD47, through its actions on both osteoblasts and osteoclasts, has a profound effect on skeletal remodeling and bone maintenance. Initial phenotypic characterization of $C d 47$ null mice showed a defect in the host defense pathway manifested as both delayed polymorphonuclear leukocyte migration to the site of infection and to defective activation at the site ${ }^{7}$. More recently, Uluckan et al. reported young $C d 47^{-1}$ mice were osteopetrotic and that trabecular BV/TV was increased ${ }^{22}$. In contrast to that study we found that $C d 47^{-1}$ males and females at 16 weeks of age have very low bone mass which results from a defect in both osteoclast fusion and osteoblastogenesis. There are two important differences that may explain this apparent discrepancy. First, in that study, all the mice were studied at one time point (i.e. 8 weeks). Second, there were only 5 animals/ genotype (in contrast to $>20$ mice/group in our study), and there was no report of gender inclusion. Based on previous in vitro studies ${ }^{10}$, we hypothesized that CD47 may be an important regulator of both bone formation and resorption, and that any perturbationin its expression would significantly affect bone maintenance. Indeed, measurements of both cortical and trabecular bone mass using areal and volumetric determinations show that absence of CD47 causes a temporal but profound change in skeletal structure which is more pronounced in females than males, and in older rather than younger mutant mice.

The skeletal changes can best be interpreted within a developmental context and a background of the cell autonomous effects noted in our in vitro studies. Although total aBMD increased progressively in both male and females regardless of genotype, the difference between $C d 47$ nulls and controls became much greater as the mice aged. These observations are supported by the dynamic histomorphometric analyses which convincingly demonstrated a nearly $50 \%$ drop in bone formation rate (BFR/TV) and a $25 \%$ drop in 
mineral apposition rate (MAR) in the male $C d 47^{-/}$compared to $C d 47^{+/+}$controls. This was associated with reduced osteoblast number and function, even in the face of little change in the number of osteoclasts. Interestingly, one measure of osteoclast function, eroded surfaces/ bone surfaces, was decreased in the $C d 47^{-/}$mice compared to controls. Although not significant, this decrease is consistent with the in vitro data that demonstrated impaired osteoclast fusion and function. Taken together these data would suggest a markedly imbalanced remodeling sequence due to suppressed bone formation and modestly reduced bone resorption.

We initially established that contrary to earlier studies, both osteoblasts and osteoclasts have cell autonomous defects in $C d 47^{-1-}$ mice. In vitro, bone marrow mesenchymal stromal cells from $C d 47^{-1}$ mice did not differentiate as well as cells from $C d 47^{+/+}$controls, and had fewer von Kossa positive colonies after 14 and 21 days in culture. These findings combined with our studies showing that CD47 is expressed in osteoblasts suggest that absence of CD47 may have a profound effect on stromal cell differentiation into the osteoblast lineage. Osteoclast fusion was also impaired in the cells derived from $\mathrm{Cd}^{-1} 7^{-}$mice such that there were very few TRAP5b positive multinucleated cells and an in vitro resorption assay demonstrated virtually no resorption of bone compared to controls. It is therefore conceivable that the primary defect in the skeleton is on the resorption side during early growth and development (i.e. 8 weeks of age), resulting in an osteopetrotic-like phenotype as reported by Uluckan et $\mathrm{al}^{22}$. Indeed in our preliminary histomorphometric analyses of 8 week female $C d 47^{--}$mice, we found slightly but not significantly greater trabecular bone volume fraction in the distal femur compared with controls (data not shown). In contrast, the progressive loss of bone between 8 and 16 weeks of age almost certainly is due to impaired bone formation. This is supported by our findings of markedly suppressed bone formation and mineralization rates in the $C d 47^{-/}$mice, and a reduction in circulating P1NP, a marker of osteoblast function. Our results derived from the analysis of osteoblast differentiation in culture support the conclusion that the defect is cell autonomous since stimulation of mixed cultures of bone marrow cells (i.e. pre-osteoblasts and osteoclast precursors) with PTH and 1,25 dihydroxyvitamin D did not alter the osteoclast phenotype even though RANKL levels were increased. Thus, we postulate that $C d 47$ null mice have low bone mass at 16 weeks of age because of defects in both resorption and formation, with the latter being more affected at the 16 week time point and that the defect in each cell type is cell autonomous.

Two prior studies have reported a role for CD47 in osteoclast formation. Uluckan et al. reported that $\mathrm{Cd}_{4} 7^{-1}$ mice had an osteoclast defect but that it could be rescued in vitro with $100 \mathrm{ng} / \mathrm{ml}$ of RANKL ${ }^{22}$. This is in contrast to our findings and also in contrast to the study by Lundberg and colleagues in which they reported a significant reduction in OC formation from bone marrow cells derived from $\mathrm{Cd}_{4} 7^{-1-}$ mice stimulated with $100 \mathrm{ng} / \mathrm{ml}$ RANKL compared with cells from wild-type mice ${ }^{10}$. Cells of the monocyte-macrophage lineage fuse to form multinucleated giant cells. The phenotype of the giant cell varies depending upon the local environment including the chemical and physical (size) nature of the agent to which the giant cells and their precursors are responding. The in vitro studies presented here provide a mechanism for that process whereby the association of CD47 with SHPS-1 is required for the final step of osteoclast maturation as the differentiated cells fuse to form a mature cell with optimal bone resorptive capacity. This conclusion is based upon our data showing that following stimulation with RANKL, markers of osteoclast differentiation were expressed in bone marrow cells from the $\mathrm{Cd}_{4} \mathrm{~T}^{-}-$cultures to an equal extent as the wildtype cultures, the total number of TRAP5 $\mathrm{b}^{+}$cells was not significantly different yet there was a marked difference in the number of TRAP5 $\mathrm{b}^{+}$multinucleated osteoclasts.

Using alveolar macrophages that assume an osteoclast-like phenotype upon fusion, Vignery and colleagues identified novel proteins involved in fusion ${ }^{23-24}$. In their elegant study using 
a panel of antibodies directed against cell surface proteins a potential role for CD47 and its binding partner SHPS-1 in cellular fusion was demonstrated ${ }^{23-24}$. Our study supports their findings that implicate CD47-SHPS-1 in cellular fusion rather than differentiation. The role of CD47 association with SHPS-1 has been studied extensively in phagocytes. The cellsurface expression of CD47 on healthy cells and its engagement with SHPS-1 has been proposed to constitute a "don't eat me" signal thereby blocking inappropriate engulfment ${ }^{9}$. When it is associated with CD47, SHPS-1 can be tyrosine phosphorylated which initiates the recruitment, via its phosphorylated cytoplasmic tail, of the SH2 domain contaning tyrosine phosphatase, SHP-1. Recently, it was determined that dephosphorylation of non-muscle myosin IIA is a major target for SHP-1 and this dephosphorylation is critical for the prevention of engulfment of healthy cells. This process requires CD47 engagement with SHPS-1 9 . Both phagocytosis and cellular fusion require significant remodeling of the cytoskeleton. Given our findings, and those of Vignery's ${ }^{24}$ that implicated CD47 and its association with SHPS-1 in cellular fusion, we considered whether a similar mechanism as that which was identified in the phagocytes was involved in the fusion of osteoclasts. Consistent with other studies we determined that in the absence of CD47 there was no detectable SHPS-1 phosphorylation, which in turn was associated with a lack of SHP-1 recruitment to SHPS-1. Furthermore, analogous to the mechanism described in phagocytosis, non-muscle myosin IIA was highly phosphorylated in the BMC cultures from the $C d 47$ null but not wild-type mice. Thus CD47/SHPS-1 association appears to play a critical role in allowing SHP-1 recruitment and activation that is necessary for its dephosphorylation of non-muscle myosin IIA and subsequent cell fusion.

Non-muscle cell myosin IIA is a member of the mammalian class II myosin family which consists of distinct isoforms expressed in skeletal, smooth and cardiac muscle as well as three non-muscle forms designated IIA, B and C. Myosin IIA has been shown to be involved in a wide variety of cellular functions including cytokinesis, cell contractility and adhesion and motility. While in this study we did not provide direct evidence that the lack of nonmuscle cell myosin II dephosphorylation accounts for the lack of fusion in the $C d 47$ null bone marrow cells, a recent study provided more direct evidence for the role of non-muscle myosin IIA ${ }^{25}$. Using over expression and RNAi to alter myosin IIA levels McMichael et al showed that down-regulation of non-muscle myosin was required for the fusion of osteoclasts. Although the mechanism by which non-muscle myosin IIA suppresses cellular fusion in osteoclasts remains unclear, Myosin IIA may provide intracellular tension or even a physical barrier that prevents fusion. Taken together with the results from Tsai et $\mathrm{al}^{9}$ these studies strongly suggest that phosphorylation state of non-muscle myosin IIA is related to its ability to prevent fusion, however further studies will be required to unravel the mechanism by which non-muscle cell myosin IIA tyrosine phosphorylation is regulating this process.

Our findings do not provide a definitive mechanism to explain how CD147/SHPS-1 association regulates osteoblast differentiation. However the results clearly show that the two calvarial osteoblast cell lines, MC3T3-E1, clones 4 and 24, which differ significantly in their ability to undergo differentiation had markedly different levels of intact CD47 and SHPS-1 phosphorylation. In the Clone 4 cells that undergo differentiation there was a significant increase in the amount of CD47 protein that could be detected between day 5 and day 10 of differentiation whereas in the Clone 24 cells that did not differentiate there was no increase in intact CD47. To demonstrate more definitively that the difference in osteoblast potential was due to the difference in presence of intact CD47 we exposed the Clone 24 cells to thrombospondin, since we had shown previously that it increased intact CD47 in smooth muscle cells ${ }^{20}$. Thrombospondin increased intact CD47 and importantly, this was associated with an increase in SHPS-1 phosphorylation. Blocking the thrombospondin induced increase in SHPS-1 phosphorylation with the anti CD47 antibody was associated with decreased 
osteoblastogenesis thus supporting the conclusion that CD47/SHPS- 1 association is essential for this event.

Our studies in smooth muscle have shown that the association of CD47/SHPS-1 and stimulation of SHPS-1 phosphorylation is required in order to activate MAP kinase ${ }^{26}$, a kinase that is required for mechanotransduction stimulation of osteoblast proliferation ${ }^{27}$. Phosphorylated SHPS-1 functions as a molecular scaffold that is required to assemble a signaling complex composed of the tyrosine phosphatase SHP-2, c-Src and p52Shc, and assembly of this complex is required for MAP kinase activation ${ }^{28}$. The results of Rangaswani et al demonstrate that in osteoblasts a complex containing SHP-2 and c-Src assembles on the phosphorylated $\beta 3$ subunit of the $a \mathrm{~V} \beta 3$ integrin in response to mechanical stimulation and that this leads to MAP kinase activation. ${ }^{29}$ Since we have shown that phosphorylation of the $\beta 3$ subunit of aV $\beta 3$ is required for SHP-2 and Src recruitment to phosphorylated SHPS-1 in SMC ${ }^{26}$ our findings in this study suggest that a similar set of signaling proteins may be recruited to SHPS-1 in osteoblasts and this may be required for MAP kinase activation.

In summary, we performed comprehensive developmental phenotyping of $C d 47^{-/}$mice and established that both osteoblast and osteoclast differentiation are impaired in vitro and in vivo. These data support the conclusion that CD47 is a multifunctional protein that plays a key role in bone acquisition and maintenance and that the mechanism by which it functions may be a part of a more general mechanism by which several types of cells respond to stress.

\section{Acknowledgments}

The authors would like to thank Dr Roland Baron for performing the histomorphometry and Dr Eric Everett for technical assistance with the osteoblast and osteoclast cultures. The authors would also like to thank Jane Maynard and Harold Coombs III for their technical expertise.

This work was funded by grants from the National Institutes of Health AR54604 and AR53853 to CJR, AG02331 to DRC and AR043618 to WGB. BEC was funded by a School of Dentistry Student Fellowship from the University of North Carolina.

\section{References}

1. Mocsai A, et al. The immunomodulatory adapter proteins DAP12 and Fc receptor gamma-chain (FcRgamma) regulate development of functional osteoclasts through the Syk tyrosine kinase. Proc Natl Acad Sci U S A. 2004; 101:6158-6163. [PubMed: 15073337]

2. Vignery A. Macrophage fusion: the making of osteoclasts and giant cells. J Exp Med. 2005; 202:337-340. [PubMed: 16061722]

3. Jiang P, Lagenaur CF, Narayanan V. Integrin-associated protein is a ligand for the P84 neural adhesion molecule. J Biol Chem. 1999; 274:559-562. [PubMed: 9872987]

4. Seiffert M, et al. Human signal-regulatory protein is expressed on normal, but not on subsets of leukemic myeloid cells and mediates cellular adhesion involving its counter receptor CD47. Blood. 1999; 94:3633-3643. [PubMed: 10572074]

5. Vernon-Wilson EF, et al. CD47 is a ligand for rat macrophage membrane signal regulatory protein SIRP (OX41) and human SIRPalpha 1. Eur J Immunol. 2000; 30:2130-2137. [PubMed: 10940903]

6. Rosales C, Gresham HD, Brown EJ. Expression of the 50-kDa integrin-associated protein on myeloid cells and erythrocytes. J Immunol. 1992; 149:2759-2764. [PubMed: 1401911]

7. Oldenborg PA, et al. Role of CD47 as a marker of self on red blood cells. Science. 2000; 288:20512054. [PubMed: 10856220]

8. Fujioka Y, et al. A novel membrane glycoprotein, SHPS-1, that binds the SH2-domain-containing protein tyrosine phosphatase SHP-2 in response to mitogens and cell adhesion. Mol Cell Biol. 1996; 16:6887-6899. [PubMed: 8943344] 
9. Tsai RK, Discher DE. Inhibition of "self" engulfment through deactivation of myosin-II at the phagocytic synapse between human cells. J Cell Biol. 2008; 180:989-1003. [PubMed: 18332220]

10. Lundberg $\mathrm{P}$, et al. Osteoclast formation is strongly reduced both in vivo and in vitro in the absence of CD47/SIRPalpha-interaction. Biochem Biophys Res Commun. 2007; 352:444-448. [PubMed: 17126807]

11. Maile LA, Clemmons DR. Integrin-associated protein binding domain of thrombospondin-1 enhances insulin-like growth factor-I receptor signaling in vascular smooth muscle cells. Circ Res. 2003; 93:925-931. [PubMed: 14563713]

12. Maile LA, et al. Glucose Regulation of Integrin-Associated Protein Cleavage Controls the Response of Vascular Smooth Muscle Cells to Insulin-Like Growth Factor-I. Mol Endocrinol. 2008; 22:1226-1237. [PubMed: 18292237]

13. Maile LA, et al. Insulin-like growth factor-I signaling in smooth muscle cells is regulated by ligand binding to the 177CYDMKTTC184 sequence of the beta3-subunit of alphaVbeta3. Mol Endocrinol. 2006; 20:405-413. [PubMed: 16195248]

14. DeMambro VE, et al. Gender-specific changes in bone turnover and skeletal architecture in igfbp-2-null mice. Endocrinology. 2008; 149:2051-2061. [PubMed: 18276763]

15. Delahunty KM, et al. Congenic mice provide in vivo evidence for a genetic locus that modulates serum insulin-like growth factor-I and bone acquisition. Endocrinology. 2006; 147:3915-3923. [PubMed: 16675518]

16. Beamer WG, et al. Genetic dissection of mouse distal chromosome 1 reveals three linked BMD QTLs with sex-dependent regulation of bone phenotypes. J Bone Miner Res. 2007; 22:1187-1196. [PubMed: 17451375]

17. Rydziel S, et al. Nephroblastoma overexpressed (Nov) inhibits osteoblastogenesis and causes osteopenia. J Biol Chem. 2007; 282:19762-19772. [PubMed: 17500060]

18. Parfitt AM, et al. Bone histomorphometry: standardization of nomenclature, symbols, and units. Report of the ASBMR Histomorphometry Nomenclature Committee. J Bone Miner Res. 1987; 2:595-610. [PubMed: 3455637]

19. Maile LA, Badley-Clarke J, Clemmons DR. The association between integrin-associated protein and SHPS-1 regulates insulin-like growth factor-I receptor signaling in vascular smooth muscle cells. Mol Biol Cell. 2003; 14:3519-3528. [PubMed: 12972543]

20. Maile LA, et al. Glucose regulation of thrombospondin and its role in the modulation of smooth muscle cell proliferation. Exp Diabetes Res. 2010; 2010

21. Wang D, et al. Isolation and characterization of MC3T3-El preosteoblast subclones with distinct in vitro and in vivo differentiation/mineralization potential. J Bone Mineral Res. 1999; 14:893-903.

22. Uluckan O, et al. CD47 regulates bone mass and tumor metastasis to bone. Cancer Res. 2009; 69:3196-3204. [PubMed: 19276363]

23. Saginario C, et al. MFR, a putative receptor mediating the fusion of macrophages. Mol Cell Biol. 1998; 18:6213-6223. [PubMed: 9774638]

24. Han X, et al. CD47, a ligand for the macrophage fusion receptor, participates in macrophage multinucleation. J Biol Chem. 2000; 275:37984-37992. [PubMed: 10964914]

25. McMichael BK, Wysolmerski RB, Lee BS. Regulated proteolysis of nonmuscle myosin IIA stimulates osteoclast fusion. J Biol Chem. 2009; 284:12266-12275. [PubMed: 19269977]

26. Ling Y, et al. Role of SHPS-1 in the regulation of insulin-like growth factor I-stimulated Shc and mitogen-activated protien kinase activation in vascular smooth muscle cells. Mol Biol Cell. 2005; 16:3353-3364. [PubMed: 15888547]

27. Papachroni K, et al. Mechanotransduction in osteoclast regulation and bone disease. Trends Mol Med. 2009; 15:208-216. [PubMed: 19362057]

28. Lieskovska J, et al. The role of Src kinase in insulin-like growth factor-depencent mitogenic signaling in vascular smooth muscle cells. J Biol Chem. 2006; 281:25041-25053. [PubMed: 16825188]

29. Rangaswami H, et al. Cyclic GMP and protein kinase G control a Src-containing mechanosome in osteoblasts. Sci Signaling. 2010; 153:ra91. 
A

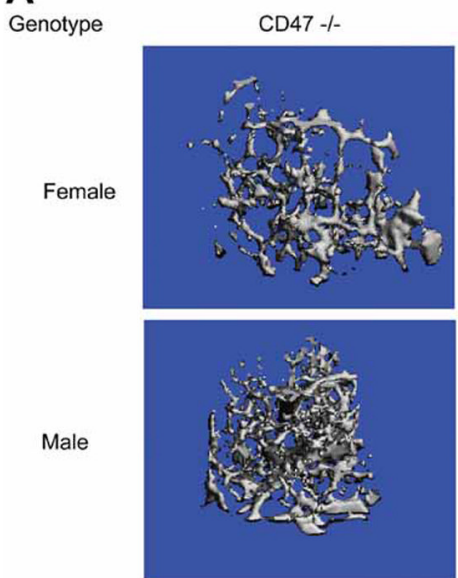

B

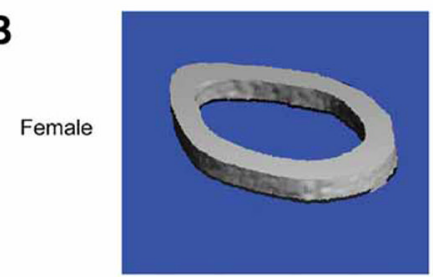

Figure 1. Micro-CT of the femur at $16 \mathrm{wk}$ of age

A: Distal trabecular compartment for the $\mathrm{Cd}_{4} \mathrm{~T}^{-1}$ and $\mathrm{Cd} 47^{+/+}$control male and females.

B: Cortical compartment for the $C d 47^{-/-}$and $C d 47^{+/+}$control male and females. 

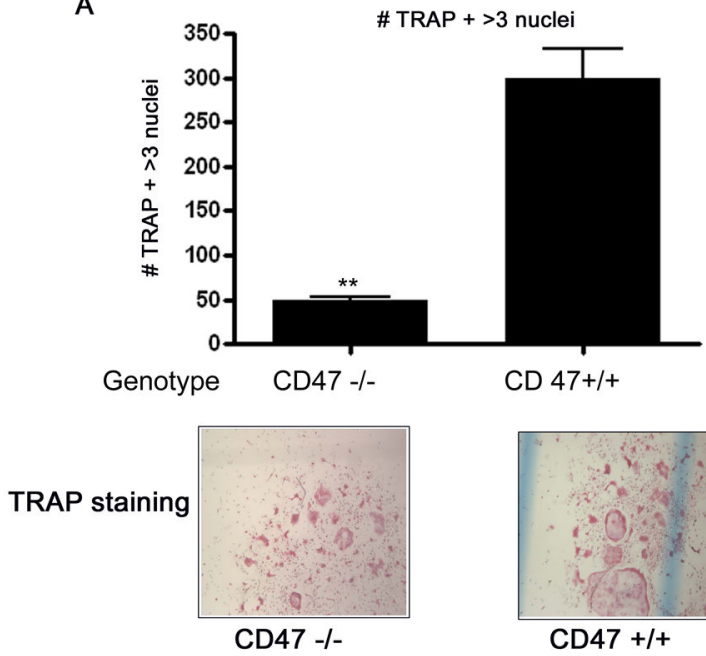

C

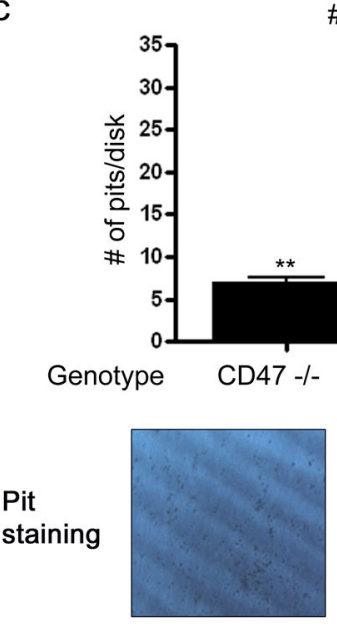

CD47 -/-

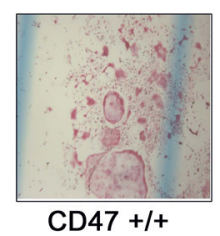

\# of pits/disk

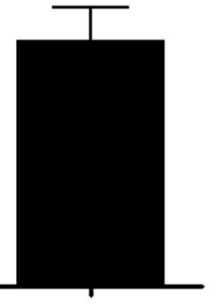

CD $47+/+$



CD47 +/+
B

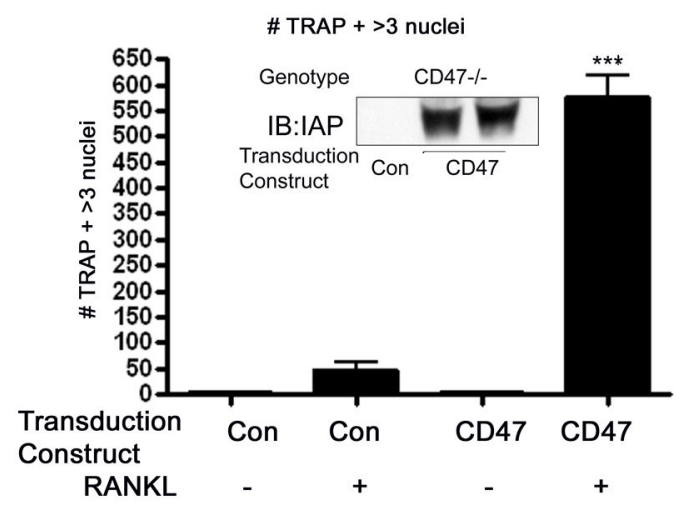

D
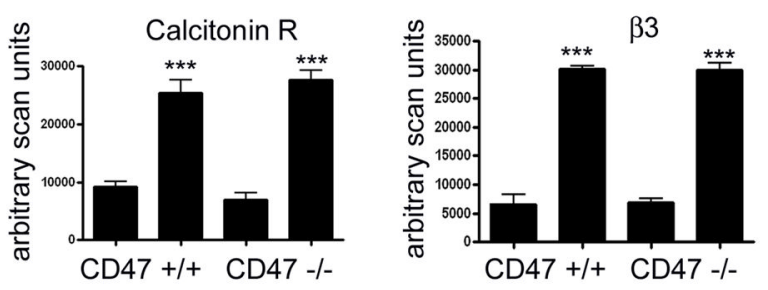

Genotype

CD47 +/+

CD47 -/-

IB:Calcitonin R

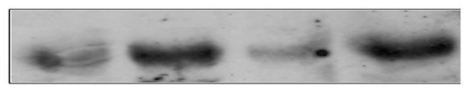

IB: $\beta 3$

IB:SHPS-1

RANKL

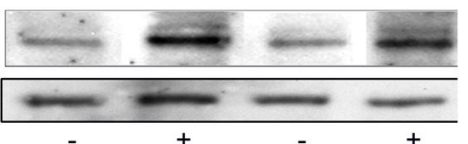

E

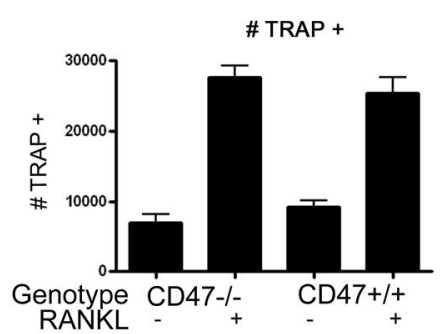

Figure 2. In vitro osteoclastogenesis

A: TRAP5b-positive (TRAP5b ${ }^{+}$) multinucleated osteoclasts in the bone marrow cultures of $C d 47^{-1-}$ and $C d 47^{+/+}$control mice. The $C d 47^{-/-}$bone marrow cells had a significant reduction in the number of osteoclast cells $(* * p<0.05)$. The graphs show the results of 5 independent experiments (upper panel).

B: Numbers of osteoclasts in the bone marrow cultures of $\mathrm{Cd}_{4} 7^{-1-}$ after transduction of either control or CD47 cDNA. The bone marrow cells expressing CD47+/+ (CD47) had a significant increase in the number of osteoclasts in response to RANKL compared to CD47 $-/-$ cultures $($ Con $)(* * * p<0.01)$. The graph shows the results of 3 independent experiments 
and the insert shows western immunoblot analysis of CD47 (IAP) protein in OC 7 days following transduction.

C: Numbers of pits in the dentin disks generated by bone marrow cultures of $\mathrm{Cd}_{4} \mathrm{~T}^{-1-}$ and $C d 47^{+/+}$control mice. The $C d 47^{-1-}$ bone marrow cells had a significant reduction in the number of pits $(* * \mathrm{p}<0.05)$. The graphs show the results of 3 independent experiments (upper panel).

D: Equal amounts of cell lysates from $C d 47^{-/}$and $C d 47^{+/+}$bone marrow cultures 7 days after stimulation with RANKL (+) in the presence of M-CSF, were separated by SDS-PAGE and immunoblotted with the indicated antibody. The graph shows the results of the immunoblots for calcitonin and $\beta 3$ derived from three similar experiments expressed as arbitrary scanning units $(* * * \mathrm{p}<0.01$ when the amount of protein in the presence of RANKL is compared with the absence of RANKL treatment). The image shown is derived from discontinuous lanes on the same blot.

E: TRAP5b-positive (TRAP5b ${ }^{+}$) osteoclasts in the bone marrow cultures of $C d 47^{-1-}$ and $C d 47^{+/+}$control mice. $* * * \mathrm{p}<0.01$ when the number of TRAP $5 b^{+}$cells in the absence of RANKL is compared with its presence and $p$, ns when the number of TRAP5b $b^{+}$cells from the $C d 47^{-1-}$ cultures is compared with the $C d 47^{+/+}$cultures. The results were obtained from 5 independent experiments. 
A

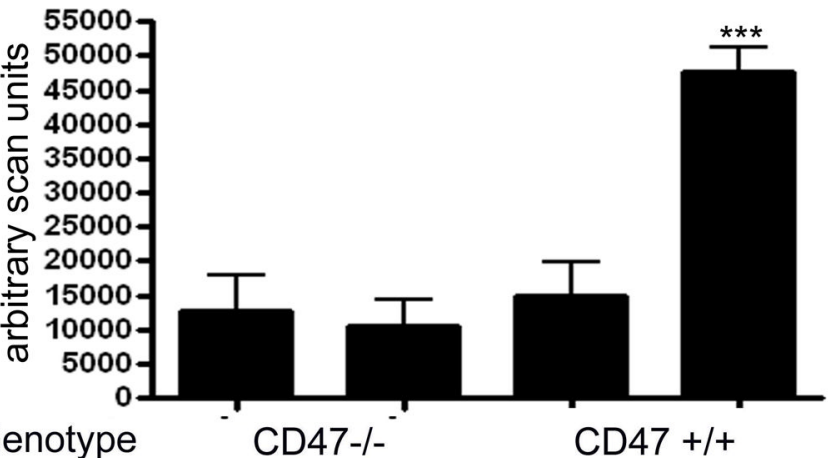

IB: CD47

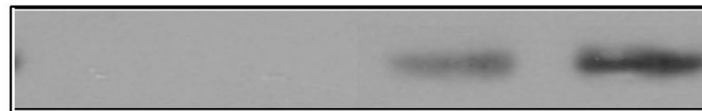

IB:SHPS-'

RANKL

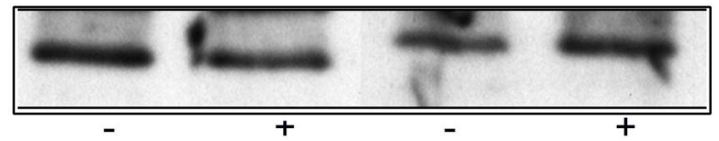

B

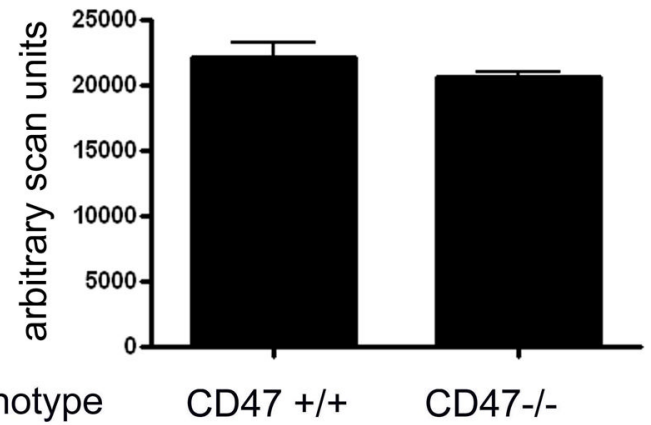

IB:RANK

RANKL

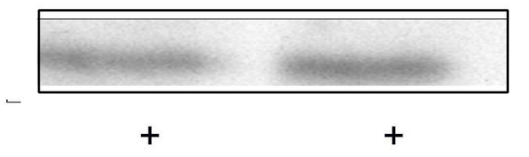

Figure 3. Western immunoblot analysis of CD47 protein levels in bone marrow cells from $\mathrm{Cd}^{-1 /-}$ and $\mathrm{Cd47^{+/+ }}$ mice

A: Equal amounts of cell lysates from $C d 47^{-/}$and $C d 47^{+/+}$bone marrow cultures 7 days after stimulation with RANKL (+) in the presence of mCSF, were separated by SDS-PAGE and immunoblotted with the indicated antibody. The graph shows the results of the CD47 immunoblots derived from three similar experiments expressed as arbitrary scanning units (*** $\mathrm{p}<0.01$ when the amount of protein in the presence of RANKL is compared with the absence of RANKL treatment). SHPS-1 is shown in the bottom panel as loading control. The image shown is derived from discontinuous lanes on the same blot.

B: Equal amounts of cell lysates from $C D 47^{-/-}$and $C D 47^{+/+}$bone marrow cultures 7 days after stimulation with RANKL (+) in the presence of M-CSF, were separated by SDS-PAGE and immunoblotted with the indicated antibody. The graph shows the results of the RANKL immunoblot derived from three similar experiments expressed as arbitrary scanning. The image shown is derived from discontinuous lanes on the same blot. 
A

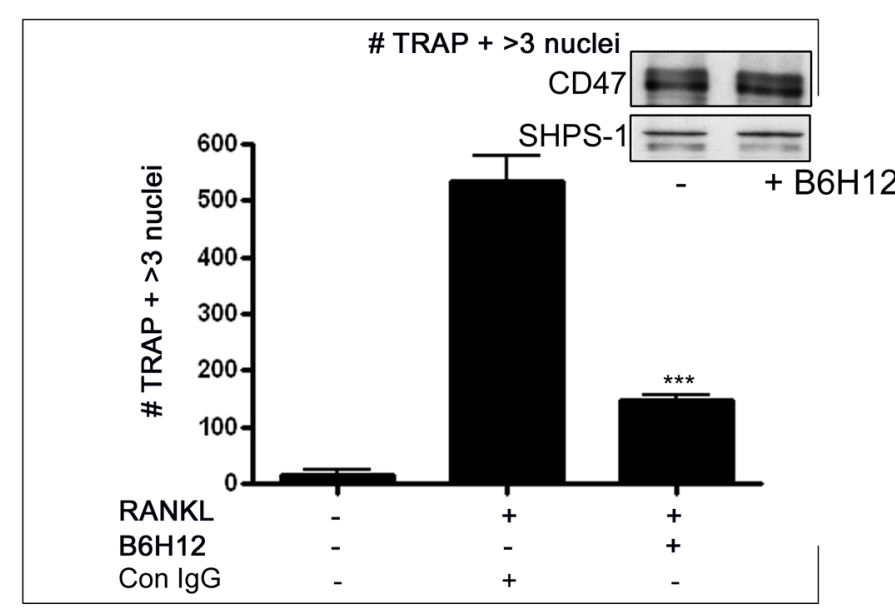

B

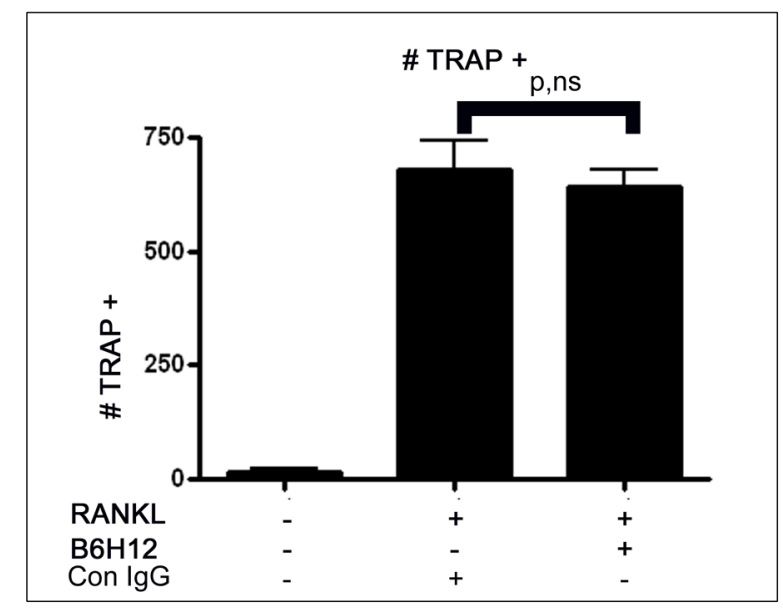

Figure 4. Effect of CD47-SHPS-1 disruption on OC formation

A: TRAP5b-positive (TRAP5b ${ }^{+}$) multinucleated (more than three nuclei) in the bone marrow cultures of $\mathrm{Cd}_{4} 7^{+/+}$mice in the presence of either control antibody (Con IgG) of the anti-CD47 antibody, B6H12. The bone marrow cells treated with B6H12 had a significant reduction in the number of TRAP5 $\mathrm{b}^{+}$multinucleated cells $(* * * \mathrm{p}<0.01)$.

B: Numbers of TRAP5 $\mathrm{b}^{+}$cells in the bone marrow cultures from $\mathrm{Cd} 47^{+/+}$mice treated with or without the anti-CD47 antibody, B6H12. p, ns when the number of TRAP5b ${ }^{+}$cells from both cultures is compared.

The graphs show the results from 3 independent experiments. 
A
Genotype
CD47 -/-
CD47+/+

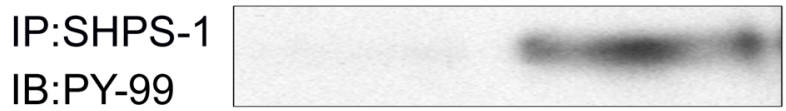

IP:SHPS-1

IB:SHP-1

IB:SHPS-1

B
RANKL

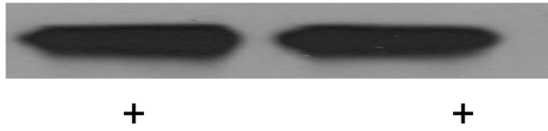

Genotype

IP:PY-99

IB:MHY-9

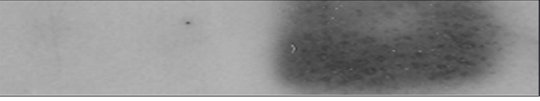

CD47 -/-

CD47+/+

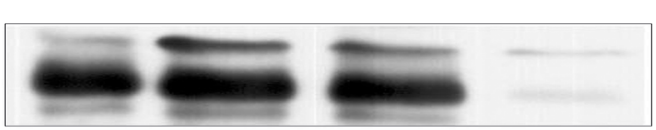

IB:SHPS-1

IB:MHY-9

RANKL

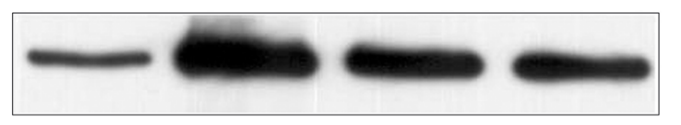
non-muscle myosin IIA phosphorylation (MHY-9) indicated antibody.
SHPS-1

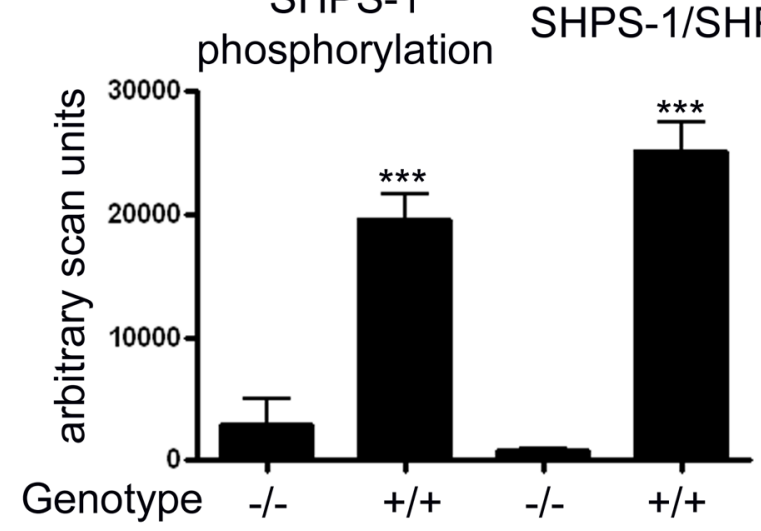

of 15000 MHY-9 Phosphorylation

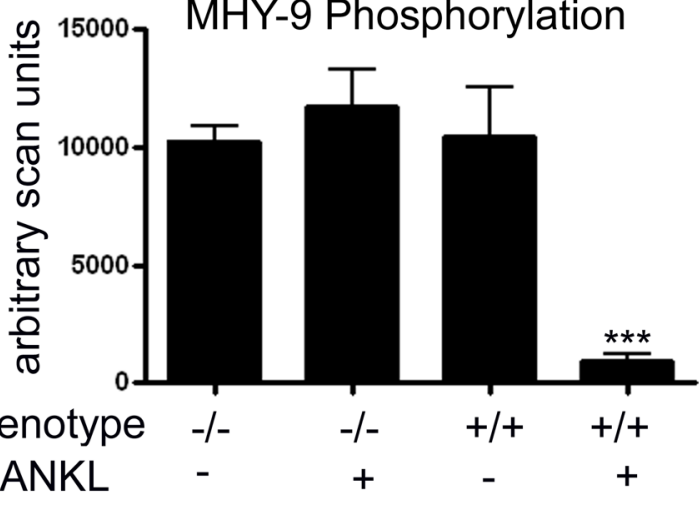

Figure 5. Western immunoblot analysis of SHPS-1 phosphorylation, SHP-1 recruitment and

A \& B: Equal amounts of cell lysates from $C d 47^{-1-}$ and $C d 47^{+/+}$bone marrow cultures that were obtained following 7 days of stimulation with RANKL (+) in the presence of M-CSF, were immunoprecipitated and then separated by SDS-PAGE and immunoblotted with the

The graphs show the results of the immunoblots derived from three similar experiments expressed as arbitrary scanning units. (*** p $<0.01$ when the amount of each phosphorylated protein in the $C d 47^{-/}$is compared with the $C d 47^{+/+}$cultures). The image shown is derived from discontinuous lanes on the same blot. Non-muscle region myosin IIA was analyzed as a loading control and showed no difference. 
A

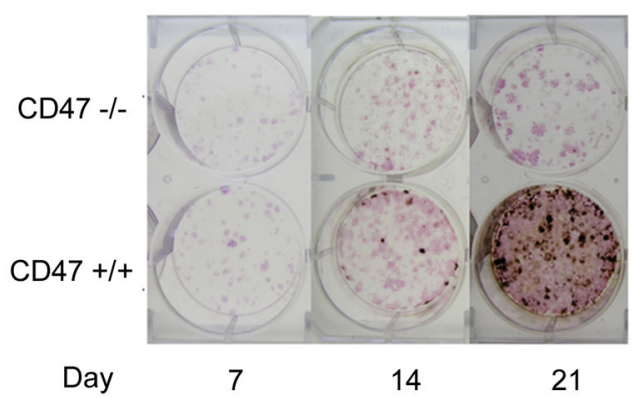

B
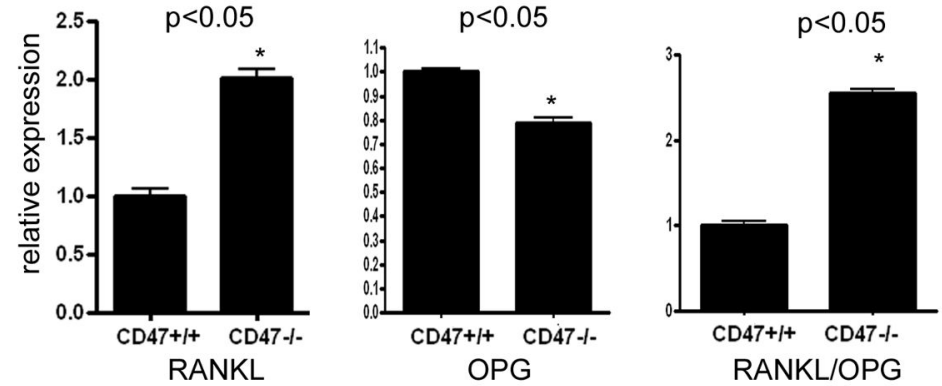

$E$

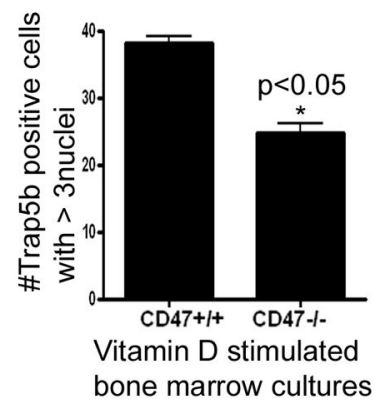

$\mathrm{F}$
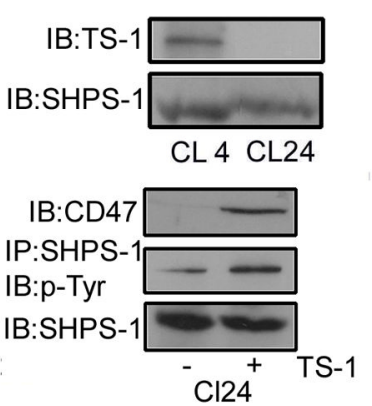

G

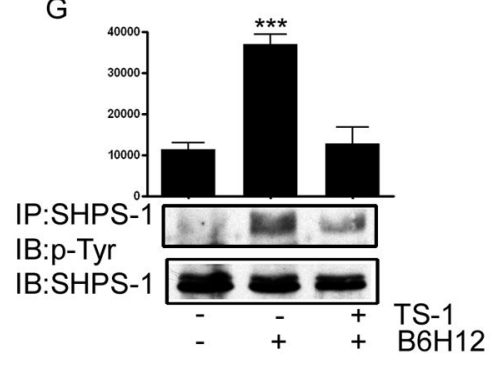

Differentiation medium MC3T3-E1 clone

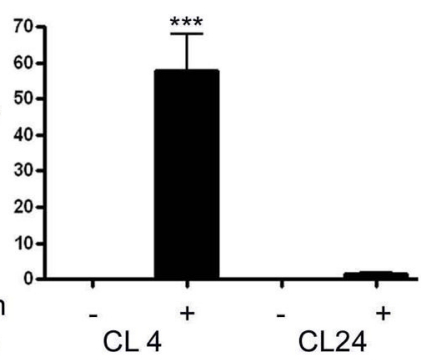

IB:SHPS- 1 days in differentiation

$\mathrm{H}$

No of Alk Phos + colonies

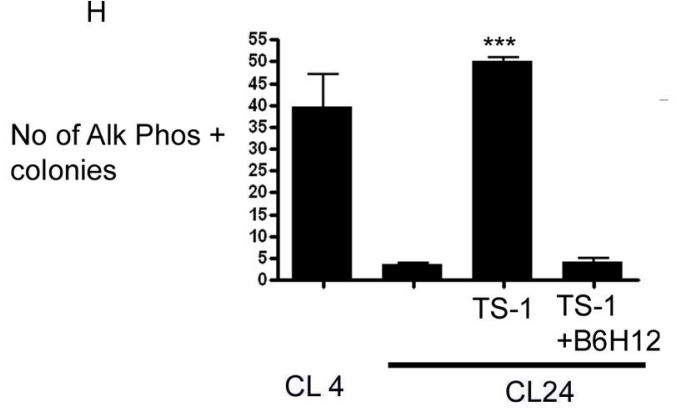

Figure 6. Analysis of a role for CD47 in osteoblast differentiation

A: At d 7, 14 and 21 in culture, adherent OB progenitor cells from bone marrow cultures were identified by alkaline phosphatase staining (preosteoblasts; CFU-AP ${ }^{+}$). At d 14 and 21 differentiated cultures were counterstained with von Kossa for mineral deposits (CFU-OB)

B: Gene expression of RANKL and OPG in d 7 cultures.

C: Trap 5b+ cells in mixed bone marrow cultures stimulated with Vitamin D.

D: Numbers of osteoblast cells in Clone 4 (CL-4) and Clone-24 (CL-24) cultures after 14 days in differentiation medium. At day 14 the cells were fixed and stained for alkaline phosphatase activity (AP). AP-positive $\left(\mathrm{AP}^{+}\right)$pre-osteoblasts were then counted using light microscopy. $* * * \mathrm{p}<0.01$ when the number of $\mathrm{AP}^{+}$cells in the CL-4 cultures is compared with the CL-24. The graph shows the results from 3 independent experiments.

E: Equal amounts of cell lysates from CL-4 and CL-24 cultures after 5 and 14 days incubation in differentiation medium were separated by SDS-PAGE and immunoblotted with the indicated antibody. The results of three separate experiments expressed as arbitrary scanning units are shown. *** $\mathrm{p}<0.01$ when the amount of intact CD47 protein in the CL-4 cultures is compared with the CL-24 cultures after 14 days in differentiation medium. 
F: Equal amounts of extracellular matrix from CL-4 and CL-24 cultures after 14 days incubation in differentiation medium were separated by SDS-PAGE and immunoblotted with an anti-TSP-1 antibody (top panel). To control for loading lysates from the same cultures were immunoblotted for SHPS-1. Equal amounts of lysates from CL-24 cultures after 14 days incubation in differentiation medium containing thrombospondin -1 (TS-1+) $(1 \mu \mathrm{g} / \mathrm{ml})$ were separated by SDS-PAGE and either immunoprecipitated (IP) or immunoblotted (IB) with antibodies indicated. As a control the same lysates were immunoblotted for SHPS-1.

G: Equal amounts of lysates from CL-24 cultures after 14 days incubation in differentiation medium containing thrombospondin -1 (TS-1+) $(1 \mu \mathrm{g} / \mathrm{ml})$ and/or B6H12 $(5 \mu \mathrm{g} / \mathrm{ml})$ were separated by SDS-PAGE and either immunoprecipitated (IP) or immunoblotted (IB) with antibodies indicated. To control for loading the same lysates were immunoblotted for SHPS-1.

H: Numbers of osteoblast cells in Clone 4 (CL-4) and Clone-24 (CL-24) cultures after 14 days in differentiation medium (containing thrombospondin -1 (TS-1) $(1 \mu \mathrm{g} / \mathrm{ml})$ and/or B6H12 $(5 \mu \mathrm{g} / \mathrm{ml})$. At day 14 the cells were fixed and stained for alkaline phosphatase activity (AP). AP-positive $\left(\mathrm{AP}^{+}\right)$pre-osteoblasts were then counted using light microscopy. *** $\mathrm{p}<0.01$ when the number of $\mathrm{AP}^{+}$cells in the CL-24 treated cultures is compared with the CL-24 control cultures. 
Table 1

Body composition measured by PIXImus in $C d 47^{+/+}$and $C d 47^{-/}$males and females at $4,8,12$ and 16 weeks of age.

\begin{tabular}{|c|c|c|c|c|}
\hline \multirow[b]{2}{*}{$4 w k$} & \multicolumn{2}{|c|}{ Male } & \multicolumn{2}{|c|}{ Female } \\
\hline & $\begin{array}{c}\mathrm{Cd} 47^{+/+} \\
\mathrm{n}=40\end{array}$ & $\begin{array}{c}\mathrm{Cd} 47^{-/-} \\
\mathrm{n}=35\end{array}$ & $\begin{array}{c}\mathrm{Cd} 47^{+/+} \\
\mathrm{n}=40\end{array}$ & $\begin{array}{c}C d 47^{-/-} \\
\mathrm{n}=46\end{array}$ \\
\hline Body Weight (g) & $14.3 \pm 0.2$ & $13.3 \pm 0.3^{a}$ & $12.6 \pm 0.2$ & $12.2 \pm 0.2$ \\
\hline $\operatorname{BMD}\left(\mathrm{g} / \mathrm{cm}^{2}\right)$ & $0.03 \pm .001$ & $0.03 \pm .001$ & $0.03 \pm .001$ & $0.03 \pm .001$ \\
\hline $\mathrm{BMC}(\mathrm{g})$ & $0.184 \pm .003$ & $0.175 \pm .004$ & $0.174 \pm .003$ & $0.170 \pm .003$ \\
\hline Lean mass (g) & $12.0 \pm 0.2$ & $11.1 \pm 0.2^{a}$ & $10.2 \pm 0.3$ & $10.1 \pm 0.2$ \\
\hline$\%$ Fat & $12.9 \pm 0.1$ & $13.3 \pm 0.1^{a}$ & $14.3 \pm 0.2$ & $14.7 \pm 0.2$ \\
\hline 8 wk & $\mathrm{n}=41$ & $\mathrm{n}=49$ & $\mathrm{n}=43$ & $\mathrm{n}=60$ \\
\hline Body Weight (g) & $23.7 \pm 0.3$ & $21.9 \pm 0.3^{a}$ & $17.6 \pm 0.3$ & $17.2 \pm 0.2$ \\
\hline $\operatorname{BMD}\left(\mathrm{g} / \mathrm{cm}^{2}\right)$ & $0.046 \pm .001$ & $0.043 \pm .001^{a}$ & $0.042 \pm .001$ & $0.041 \pm .001^{a}$ \\
\hline $\mathrm{BMC}(\mathrm{g})$ & $0.431 \pm .006$ & $0.393 \pm .006^{a}$ & $0.356 \pm .006$ & $0.337 \pm .005^{a}$ \\
\hline Lean mass (g) & $19.9 \pm 0.3$ & $18.1 \pm 0.2^{a}$ & $14.1 \pm 0.3$ & $13.8 \pm 0.2$ \\
\hline$\%$ Fat & $14.1 \pm 0.3$ & $14.6 \pm 0.3$ & $16.3 \pm 0.3$ & $16.6 \pm 0.3$ \\
\hline $12 \mathrm{wk}$ & $\mathrm{n}=38$ & $n=33$ & $\mathrm{n}=28$ & $\mathrm{n}=39$ \\
\hline Body Weight (g) & $27.1 \pm 0.3$ & $24.4 \pm 0.3^{a}$ & $20.9 \pm 0.3$ & $19.7 \pm 0.2^{a}$ \\
\hline $\operatorname{BMD}\left(\mathrm{g} / \mathrm{cm}^{2}\right)$ & $0.049 \pm .001$ & $0.045 \pm .001^{a}$ & $0.046 \pm .001$ & $0.044 \pm .001^{a}$ \\
\hline $\mathrm{BMC}(\mathrm{g})$ & $0.502 \pm .009$ & $0.441 \pm .007^{a}$ & $0.440 \pm .006$ & $0.410 \pm .006^{a}$ \\
\hline Lean mass (g) & $22.5 \pm 0.3$ & $20.2 \pm 0.3^{a}$ & $17.3 \pm 0.2$ & $15.9 \pm 0.2^{a}$ \\
\hline$\%$ Fat & $14.7 \pm 0.4$ & $14.9 \pm 0.3$ & $14.6 \pm 0.4$ & $15.7 \pm 0.4$ \\
\hline $16 \mathrm{wk}$ & $\mathrm{n}=38$ & $\mathrm{n}=40$ & $\mathrm{n}=21$ & $\mathrm{n}=37$ \\
\hline Body Weight (g) & $25.3 \pm 0.3$ & $24.1 \pm 0.4^{a}$ & $18.4 \pm 0.3$ & $18.1 \pm 0.3$ \\
\hline $\operatorname{BMD}\left(\mathrm{g} / \mathrm{cm}^{2}\right)$ & $0.051 \pm .001$ & $0.048 \pm .001^{a}$ & $0.049 \pm .001$ & $0.047 \pm .001^{a}$ \\
\hline BMC (g) & $0.501 \pm .010$ & $0.461 \pm .007^{a}$ & $0.450 \pm .005$ & $0.427 \pm .007^{a}$ \\
\hline Lean mass $(\mathrm{g})$ & $19.2 \pm 0.4$ & $18.6 \pm 0.3$ & $14.0 \pm 0.2$ & $14.0 \pm 0.3$ \\
\hline$\%$ Fat & $20.5 \pm 0.7$ & $19.2 \pm 0.5$ & $19.7 \pm 0.6$ & $18.6 \pm 0.5$ \\
\hline
\end{tabular}

$\stackrel{a}{=} \mathrm{p}<.05$ 
Table 2

Femoral pQCT phenotypes for $C d 47^{+/+}$and $C d 47^{-/-}$females and males at 8 and 16 wks of age.

\begin{tabular}{|c|c|c|c|c|}
\hline \multirow[b]{2}{*}{8 weeks } & \multicolumn{2}{|c|}{ Male } & \multicolumn{2}{|c|}{ Female } \\
\hline & $\begin{array}{c}\mathrm{Cd} 47^{+/+} \\
\mathrm{n}=17\end{array}$ & $\begin{array}{c}\mathrm{Cd} 47^{-/-} \\
\mathrm{n}=12\end{array}$ & $\begin{array}{c}C d 47^{+/+} \\
\mathrm{n}=13\end{array}$ & $\begin{array}{c}C d 47^{-/-} \\
\mathrm{n}=13\end{array}$ \\
\hline Femur Length (mm) & $15.03 \pm 0.08$ & $14.62 \pm 0.14^{b}$ & $14.42 \pm 0.06$ & $14.10 \pm 0.06^{b}$ \\
\hline total vBMD $\left(\mathrm{mg} / \mathrm{mm}^{3}\right)$ & $0.542 \pm 0.007$ & $0.490 \pm 0.015^{b}$ & $0.502 \pm 0.006$ & $0.448 \pm 0.006^{a}$ \\
\hline cortical vBMD $\left(\mathrm{mg} / \mathrm{mm}^{3}\right)$ & $1.005 \pm 0.005$ & $0.996 \pm 0.004$ & $1.000 \pm 0.005$ & $0.982 \pm 0.004^{b}$ \\
\hline Cortical Th. (mm) & $0.190 \pm 0.005$ & $0.161 \pm 0.006^{b}$ & $0.153 \pm 0.002$ & $0.139 \pm 0.002^{a}$ \\
\hline Peri C (mm) & $5.242 \pm 0.068$ & $4.923 \pm 0.046^{b}$ & $4.79 \pm 0.04$ & $4.69 \pm 0.03^{b}$ \\
\hline Endo C (mm) & $4.051 \pm 0.048$ & $3.912 \pm 0.031^{b}$ & $3.824 \pm 0.042$ & $3.814 \pm 0.032$ \\
\hline 16 weeks & $\mathrm{n}=28$ & $\mathrm{n}=33$ & $\mathrm{n}=20$ & $\mathrm{n}=20$ \\
\hline Femur Length (mm) & $16.05 \pm 0.07$ & $15.64 \pm 0.06^{a}$ & $15.57 \pm 0.06$ & $15.33 \pm 0.09^{b}$ \\
\hline total vBMD $\left(\mathrm{mg} / \mathrm{mm}^{3}\right)$ & $0.571 \pm 0.006$ & $0.526 \pm 0.005^{a}$ & $0.554 \pm 0.004$ & $0.520 \pm 0.006^{a}$ \\
\hline cortical vBMD $\left(\mathrm{mg} / \mathrm{mm}^{3}\right)$ & $1.072 \pm 0.003$ & $1.059 \pm 0.003^{b}$ & $1.082 \pm 0.004$ & $1.068 \pm 0.005^{b}$ \\
\hline Cortical Th. (mm) & $0.187 \pm 0.003$ & $0.169 \pm 0.001^{a}$ & $0.179 \pm 0.002$ & $0.166 \pm 0.002^{a}$ \\
\hline Peri C (mm) & $5.482 \pm 0.062$ & $5.122 \pm 0.044^{a}$ & $4.91 \pm 0.04$ & $4.90 \pm 0.03$ \\
\hline Endo C (mm) & $4.306 \pm 0.055$ & $4.059 \pm 0.040^{b}$ & $3.782 \pm 0.033$ & $3.856 \pm 0.031$ \\
\hline
\end{tabular}


Table 3

Femoral MicroCT analysis of $\mathrm{Cd} 47^{+/+}$and $C d 47^{-/-}$mice at 16 weeks of age.

\begin{tabular}{|c|c|c|c|c|}
\hline & \multicolumn{2}{|c|}{ Male } & \multicolumn{2}{|c|}{ Female } \\
\hline & $\begin{array}{c}\mathrm{Cd} 47^{+/+} \\
\mathrm{n}=15\end{array}$ & $\begin{array}{c}C d 47^{-/-} \\
\mathrm{n}=15\end{array}$ & $\begin{array}{c}\mathrm{Cd} 47^{+/+} \\
\mathrm{n}=15\end{array}$ & $\underset{\mathrm{n}=15}{\mathrm{Cd} 47^{-/-}}$ \\
\hline \multicolumn{5}{|l|}{ Midshaft } \\
\hline$\% \mathrm{BA} / \mathrm{TA}$ & $46.2 \pm 3.0$ & $45.7 \pm 3.0$ & $48.2 \pm 3.0$ & $45.4 \pm 3.0^{a}$ \\
\hline Ct. Th $(\mu \mathrm{m})$ & $208.6 \pm 2.6$ & $199.2 \pm 2.6^{b}$ & $200.9 \pm 2.1$ & $185.1 \pm 2.1^{b}$ \\
\hline \multicolumn{5}{|l|}{ Distal } \\
\hline$\% \mathrm{BV} / \mathrm{TV}$ & $16.1 \pm 4.0$ & $12.4 \pm 0.9^{b}$ & $5.1 \pm 0.3$ & $4.0 \pm 0.4^{b}$ \\
\hline Tb. N (1/mm) & $5.01 \pm 0.08$ & $4.70 \pm 0.08^{b}$ & $3.71 \pm 0.06$ & $3.48 \pm 0.06^{b}$ \\
\hline Tb. Th $(\mu \mathrm{m})$ & $50.1 \pm 0.7$ & $48.1 \pm 0.7$ & $36.9 \pm 0.7$ & $34.8 \pm 0.7^{b}$ \\
\hline $\mathrm{Tb} . \mathrm{Sp}(\mu \mathrm{m})$ & $193.4 \pm 1.9$ & $209.5 \pm 2.9^{b}$ & $270.2 \pm 5.0$ & $289.7 \pm 5.0^{b}$ \\
\hline
\end{tabular}

$\stackrel{a}{=} \mathrm{p} \leq 0001$

$b^{b} \mathrm{p} \leq 05$ 
Table 4

Histomorphometric analysis of 16 week old $C d 47^{+/+}$and $C d 47^{-/-}$male femurs

\begin{tabular}{|c|c|c|}
\hline Parameters & $\begin{array}{r}C d 47^{+/+} \\
\mathrm{n}=7\end{array}$ & $\begin{array}{c}\mathrm{Cd}_{\mathrm{n}=5}{ }^{-/-} \\
\end{array}$ \\
\hline $\mathrm{BV} / \mathrm{TV}(\%)$ & $10.03 \pm 0.64$ & $8.43 \pm 0.94$ \\
\hline MS/BS (\%) & $49.35 \pm 3.01$ & $38.95 \pm 1.14^{a}$ \\
\hline MAR (mm/day) & $1.04 \pm 0.05$ & $0.75 \pm 0.05^{b}$ \\
\hline $\mathrm{BFR} / \mathrm{BS}\left(\mathrm{mm}^{3} / \mathrm{mm}^{2} /\right.$ year$)$ & $187.0 \pm 14.0$ & $106.0 \pm 6.0^{b}$ \\
\hline N.Ob/B.Pm (/mm) & $3.61 \pm 0.52$ & $1.31 \pm 0.12^{b}$ \\
\hline OS/BS (\%) & $1.76 \pm 0.25$ & $0.34 \pm 0.14^{b}$ \\
\hline O.Th (mm) & $2.62 \pm 0.18$ & $1.03 \pm 0.47^{b}$ \\
\hline N.Oc/B.Pm $(/ \mathrm{mm})$ & $0.61 \pm 0.12$ & $0.64 \pm 0.15$ \\
\hline ES/BS (\%) & $0.91 \pm 0.33$ & $0.53 \pm 0.28$ \\
\hline
\end{tabular}

$\stackrel{a}{=} p<0.05$

$b^{b}=p<0.01$ 
Table 5

Analysis of Serum markers for bone turnover in $C d 47^{+/+}$and $C d 47^{-/-}$males at 8, 12 and 16 weeks of age.

\begin{tabular}{ccc}
\hline & $\boldsymbol{C d \boldsymbol { d A } ^ { + / + } \text { Males }}$ & $\boldsymbol{C d \boldsymbol { d }}^{-/-}$Males \\
\hline 8wk & $\mathrm{n}=10$ & $\mathrm{n}=13$ \\
IGF-1 & $325.1 \pm 10.3$ & $248.9 \pm 4.5^{a}$ \\
$12 \mathrm{wk}$ & $\mathrm{n}=10$ & $\mathrm{n}=10$ \\
PINP & $28.56 \pm 1.28$ & $23.71 \pm 1.61^{b}$ \\
$\beta$-CTx & $15.87 \pm 0.98$ & $16.03 \pm 1.23$ \\
16 wk & $\mathrm{n}=15$ & $\mathrm{n}=12$ \\
IGF-1 & $266.7 \pm 4.9$ & $283.9 \pm 6.8$ \\
MOC & $73.7 \pm 4.3$ & $70.6 \pm 3.5$ \\
TRAP5b & $10.9 \pm 1.0$ & $12.5 \pm 1.4$ \\
\hline
\end{tabular}

$\stackrel{a}{=} \mathrm{p} \leq 0001$

$b^{b} \mathrm{p} \leq 05$ 